\title{
A Voluntary Relaying MAC Protocol for Multi-rate Wireless Local Area Networks ${ }^{\star}$
}

\author{
Jaeeun Na, Yeonkwon Jeong, and Joongsoo Ma \\ School of Engineering, Information and Communications University \\ Daejeon, 305-732, Korea \\ \{davini02, ykwjeong, jsma\}@icu.ac.kr
}

\begin{abstract}
To exploit multi-rate capability as well as improve performance in wireless local area networks (WLANs), many mechanisms were proposed on IEEE 802.11 media access control (MAC) layer. However, no effort has been invested to exploit the multi-rate capability for power saving mechanism in MAC layer. In this paper, we propose a Voluntary Relaying MAC Protocol, called VRMP, to achieve both performance improvement and power saving by leveraging the multi-rate capability. In voluntary relaying scheme, if a node can support low rate node's data packet at higher rate and has sufficient power, after cooperatively sending data packet at higher rate, all nodes go into sleep mode as quickly as possible to reduce power consumption. Simulation results show that the VRMP improves throughput by $30 \sim 60 \%$ as well as reduces power consumption by $10 \sim 55 \%$ than the legacy mechanism.
\end{abstract}

\section{Introduction}

IEEE 802.11 standard for wireless LAN [1] provides a multi-rate capability on physical layer (PHY) to support higher bandwidth by using different modulation schemes. For example, IEEE 802.11b supports data rates of 1, 2, 5.5, 11Mbps, which are inversely proportional with the transmission distance between sender and receiver. To improve performance by exploiting multi-rate capability on medium access control (MAC) layer [2, many relaying MAC protocols [3, 4, 5] were proposed recently. This approach makes that low rate transmission is replaced with two higher rate transmissions by using intermediate node as relay node. However, these solutions only consider how to transmit data packet at higher rate through relay node between sender and receiver. In addition, low rate node selects a relay node without regard to mobility and power status of relay node. In some case, relay node may consume more battery power due to packet transmission of other nodes. Moreover, as the number of nodes increase, the overheads by overhearing other node's packets and maintenance of table are proportionally increased. Hence, this paper proposes a Voluntary Relaying

\footnotetext{
* This research was supported by MIC (Ministry of Information and Communication), Korea, under the ITRC (Information Technology Research Center) support program supervised by the IITA (Institute of Information Technology Assessment). (IITA2006-C1090-0603-0015).
} 


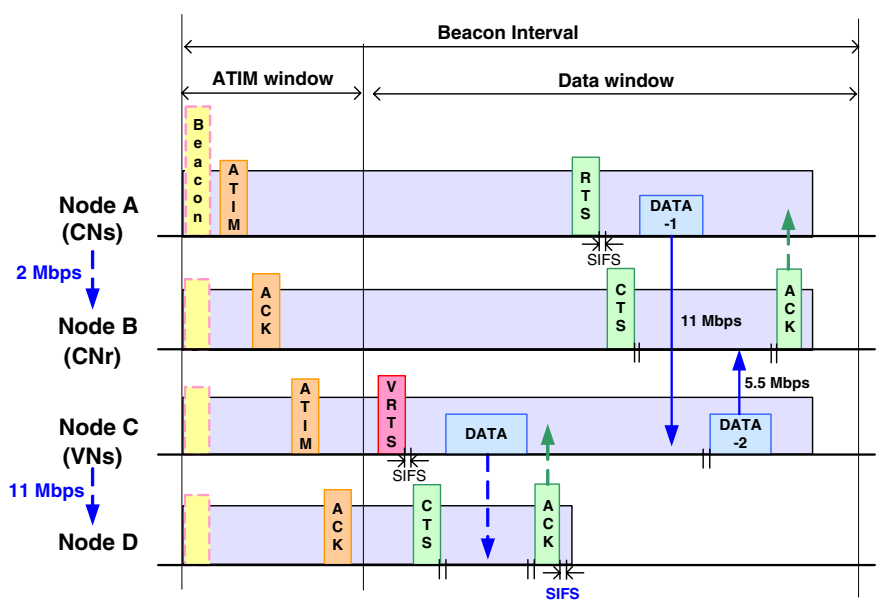

Fig. 1. The Operation of VRMP

MAC Protocol (VRMP) that leverages the multi-rate capability with power saving mode (Section 2). In addition, in VRMP, relay node notifies other low rate nodes voluntarily that allows indirect data transmission via itself. After relaying data transmission, nodes enter into doze mode to save unnecessary power. Thus, VRMP not only increases network throughput, but also reduces average power consumption (Section 3).

\section{The Voluntary Relaying MAC Protocol}

In this section, we propose the Voluntary Relaying MAC protocol (VRMP) that is based on power saving mechanism (PSM) of 802.11b. The basic concept of VRMP is that every awake node quickly enters doze mode through cooperatively helping transmit data packet at higher rate. To save unnecessary idle power of legacy PSM, sender or receiver can go into doze mode when they have no more sending or receiving data packet, including other node's relaying data packet. Each node also maintains the candidate table only for current beacon interval to assist low rate transmission of other node. In addition, we restrict that a node can assist only one neighbor node to avoid much power consumption by relaying many other data packets.

The VRMP operation is illustrated in Figure 1, and is explained in detail by the following steps:

1) In ATIM (Announcement Traffic Indication Message) window, sender transmits ATIM frame and receiver decides direct rate $\left(R_{d}\right)$ by measuring its received signal strength. And then, receiver notifies it to sender and neighbor nodes via ACK (Acknowledgement) frame. Every node overhears all ongoing ATIM/ACK frames and determines two indirect rates between sender (or receiver) and itself 
respectively. If $R_{d}$ is lower than $2 \mathrm{Mbps}$ and it supports higher indirect rate than $R_{d}$, it adds the new candidate node $\left(C N_{s}\right)$ information in candidate table.

2) When ATIM window is over, a node checks whether its data packet will transmit at high rate, it has sufficient power and has more than one $C N_{s}$ in table. If these conditions are satisfied, this node enables to relay other packets, which called the voluntary node $(V N)$. They have high priority for data transmission with smaller contention window size. Since these nodes have to notify their $C N_{s}$ that they will relay low rate data packet at higher rate, before $C N_{s}$ sends out its data packet at low rate.

3 ) If the voluntary node is sender $\left(V N_{s}\right)$, it selects an optimal $C N_{s}$ with the highest indirect rates and transmits the Voluntary RTS (VRTS) frame that piggybacks helping $C N_{s}$ 's address and supporting indirect rates in RTS (Request To Send) frame. In case of receiver $\left(V N_{r}\right)$, it transmits VCTS (Voluntary Clear To Send) frame only after it receives RTS frame from its sender. At this time, if selected $C N_{s}$ overhears VRTS or VCTS frame, it prepares data transmission regardless of its remaining backoff time. Next, voluntary node begins own data transmission with its counterpart node. After that, $C N_{s}$ also starts immediately relaying transmission of own data packet via voluntary node without contention. Finally, voluntary node and candidate node go into doze mode only if there is no remaining data transmission or reception.

\section{Simulation Results}

In this section, we evaluate the performance of VRMP through simulation. Similar to [5], the distance threshold for $11,5.5,2 \mathrm{Mbps}$ are $100 \mathrm{~m}, 200 \mathrm{~m}$, and $250 \mathrm{~m}$, respectively. The data packet length is fixed at 512 bytes. The nodes are randomly distributed in $250 \mathrm{~m} \times 250 \mathrm{~m}$. The ATIM window size is 20 or $30 \mathrm{~ms}$ and beacon interval is $100 \mathrm{~ms}$. For calculating power consumption, we use $1.65 \mathrm{~W}$, $1.4 \mathrm{~W}, 1.15 \mathrm{~W}$ and $0.045 \mathrm{~W}$ as value of power consumed by MAC layer in transmit, receive, and idle modes and doze state, respectively.

Figure 2 shows the aggregate throughput during one beacon interval when using legacy PSM, VRMP. Comparing VRMP with legacy PSM, they have same throughput due to enough data window time to transmit all data packets of senders when the number of nodes is small. Otherwise, VRMP outperforms legacy PSM $(30 \% \sim 60 \%)$ when the number of nodes is more than 30 due to higher data rate transmission and voluntary relaying procedure.

Figure 3 compares the average power consumption of a node. VRMP saves power at almost 10\% 55\% than PSM because nodes enter into doze mode rapidly once they transmit or receive all packets and relaying packet. On the contrary, in PSM, all nodes must awake to transmit data packet during entire beacon interval. At VRMP with 20ms, power consumption is reduced when there is more than 50 nodes. Since many nodes cannot send ATIM frame during short ATIM window, so most of them go to sleep mode until next beacon interval. 


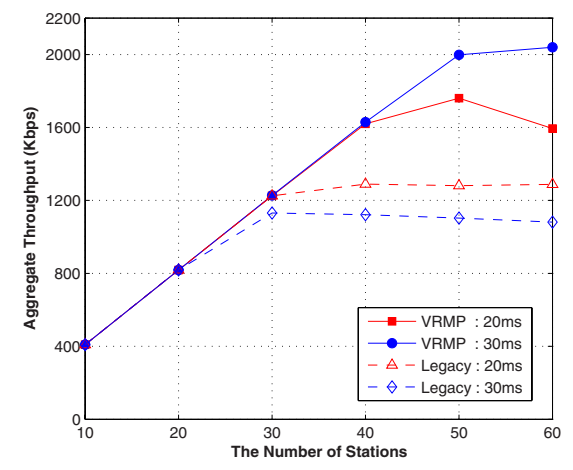

Fig. 2. Aggregate Throughput

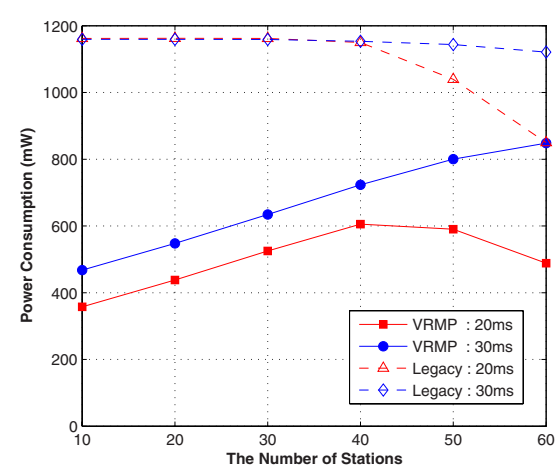

Fig. 3. Power Consumption

However, it may lead to longer transmission delay. In case of VRMP with 30ms, it results the higher power consumption than $20 \mathrm{~ms}$ because all nodes must maintain awake state for longer duration of ATIM window.

\section{Conclusion}

In this paper, we propose the voluntary relaying MAC protocol that exploits the multi-rate capability with power saving mechanism for WLANs. The neighbor nodes help voluntarily low rate node to be delivered data packet faster through indirect transmission. It also makes that all nodes can enter quickly into doze mode through voluntarily helping transmit data packet. Simulation results show that the proposed scheme outperforms the legacy PSM in terms of throughput, and power consumption. The proposed mechanism does not need a complex procedure for relaying data transmission and can be applied to mobile environments and $802.11 \mathrm{a} / \mathrm{b} / \mathrm{g}$.

\section{References}

1. "IEEE Std. 802.11b-1999, Part 11: Wireless LAN Medium Access Control (MAC) and Physical Layer (PHY) Specifications: High-Speed Physical Layer Extension in the $2.4 \mathrm{GHz}$ Band," 1999.

2. M. Heusse, F. Rousseau, G. Berger-Sabbatel, and A. Duda, "Performance anomaly of 802.11b," in Proc. of IEEE INFOCOM, San Francisco, USA, March 2003.

3. P. Liu, Z. Tao, and S. S. Panwar, "A Cooperative MAC Protocol for Wireless Local Area Networks," in Proc. of the 2005 IEEE International Conference on Communications (ICC), Seoul, Korea, May 2005.

4. L.M. Feeney, D. Hollos, H. Karl, M. Kubisch, and S. Mengesha, "A geometric derivation of the probability of finding a relay in multi-rate networks," in Proc. of 3rd IFIP-TC6 Networking Conference (Networking 2004), Athens, Greece, May 2004.

5. H. Zhu and G. Cao, "rDCF: A relay-enabled medium access control protocol for wireless ad hoc networks," in Proc. of IEEE INFOCOM, Miami, FL, Mar. 2005. 